\title{
Multi-media Educational Tool Increases Knowledge of Clinical Trials in Uganda
}

\author{
Barbara Castelnuovo', Kevin Newell2, Yukari C Manabe ${ }^{1,3}$ and Gavin Robertson ${ }^{1,4 *}$ \\ ${ }^{1}$ Infectious Diseases Institute, Makerere College of Health Sciences, Kampala, Uganda \\ ${ }^{2}$ Clinical Research Directorate/Clinical Monitoring Research Program, Leidos Biomedical Research, Inc. (formerly SAIC-Frederick Inc.), Frederick National Laboratory for \\ Cancer Research, USA \\ ${ }^{3}$ Division of Infectious Diseases, Department of Medicine, Johns Hopkins University School of Medicine, Baltimore, USA \\ ${ }^{4}$ Aeras (Current affiliation), Cape Town, South Africa
}

\begin{abstract}
Background: Informed consent is premised on the participants' understanding the scope of the research and the associated risks and benefits. . The objective was to evaluate the improvement in knowledge in a population unfamiliar with clinical trial concepts about "what it means to be part of a clinical trial" using an innovative educational tool called the 'Speaking Book'.
\end{abstract}

Methods: This was a randomized controlled trial conducted at a research site in Uganda. 201 participants were randomized to: (1) clinical trials information session control arm, or (2) clinical trials information session followed by instruction in the use of the Speaking Book with a take-home copy (intervention arm). After the session, participants of both groups completed a 22-item multiple-choice test on the rights and responsibilities of participants. Participants returned after one week to complete the same test to assess knowledge retention. The mean pre- and post-test score difference was assessed according to trial arm using an unpaired t-test of proportions.

Results: Ninety-one (90\%) participants completed both the initial and follow-up tests in the control arm and 100 $(100 \%)$ in the intervention arm. The average age of participants was 38 years, $53 \%$ were female and $67 \%$ were employed; $20 \%$ had previously been invited to participate in a clinical trial; of these, $19 \%$ had participated. The mean difference in proportion of correct responses from test 1 to test 2 was $2.7 \%(95 \% \mathrm{Cl} 0.3-5.0 \%)$ for the control arm and $11.6 \%(95 \% \mathrm{Cl} 9.3-13.7 \%)$ for the intervention arm (t-score $=-5.3$, $p$-value $<0.0001)$.

Conclusion: Participants who had instruction in the use of the Speaking Book had a larger increase in knowledge than those who had no access to this tool. To better engage patients unfamiliar with clinical trial concepts, innovative educational techniques can assist to increase knowledge to make an informed decision about participation in a clinical trial.

Keywords: Clinical trials; Informed consent; Speaking book

\section{Background}

In the twentieth century, a participant's informed consent became the backbone of ensuring ethical participation in a clinical trial. The key elements of the informed consent are: the provision of information about the research, the understanding of the information that is passed on, and the free agreement by the patients to participate in the study [1]. Research participants should be informed about the purpose of the research, the study procedures, the risks and the benefits of such procedures; the participant should also be informed regarding alternative options and the extent to which confidentiality will be maintained. Many of the precautions and considerations involved in ethical conduct rest on the basic foundation of informed consent. However, with conventional informed consent procedures, it has been observed that patients often misunderstand or forget basic practical information regarding the trials in which they participate [2,3]. It is important to note too, that the consent procedure alone does not necessarily ensure that research participants have obtained sufficient knowledge to make an informed choice about participation [4], and that limitations specific to populations with low literacy levels have been identified [5].

A number of studies have found low levels of understanding in terms of what constitutes a clinical trial and details on participation. For example, one study found that only $28 \%$ of participants knew the study's aim [4] while in another, $88 \%$ of women reported that they felt that trial participation was mandatory [6]. There appears to be a need for better ways of presenting information about clinical trials to enable research participants to make an informed decision. Various methods of improving patient knowledge and understanding of clinical trials used during the informed consent process have been evaluated, such as discussion groups, booklets and videotapes, "teach back" methods, educational modules to discuss research terminology, and audio/visual presentations [7-12]. The success of these approaches often depends on literacy level.

In a meta-analysis by Flory and Emanuel of 12 trials of multimedia interventions, all but one intervention failed to improve the participant's understanding of the clinical trial [13]. The one trial which showed efficacy had a small sample size and used a computerized presentation of information for participants who were primarily mentally ill [14]. The authors concluded that multimedia and enhanced consent forms had a limited impact on participant understanding and targeted individualized education was preferable. Another recent study of a video intervention corroborated this finding [15]. Two recent publications on a targeted educational session and a video intervention to increase participant's understanding of informed consent without the details

*Corresponding author: Gavin Robertson, Infectious Diseases Institute Makerere College of Health Sciences, Kampala, Uganda, Tel: +27 78508 1791; E-mail: grobertson@aeras.org

Received October 13, 2013; Accepted December 17, 2013; Published January 02, 2014

Citation: Castelnuovo B, Newell K, Manabe YC, Robertson G (2014) Multi-media Educational Tool Increases Knowledge of Clinical Trials in Uganda. J Clin Res Bioeth 5: 165. doi:10.4172/2155-9627.1000165

Copyright: $(2014$ Castelnuovo B, et al. This is an open-access article distributed under the terms of the Creative Commons Attribution License, which permits unrestricted use, distribution, and reproduction in any medium, provided the original author and source are credited. 
of a particular clinical trial did show improved post-training scores in addition to retention of this information $[16,17]$.

Research initiatives driven by both external and local investigators are rapidly increasing in countries within Sub-Saharan Africa where the familiarity with clinical trial concepts is generally low. Potential risks in conducting research in these environments are increased vulnerability to research exploitation and abuse but also low compliance to the study procedures, which can include low adherence to medication schedules. Educating people who are unfamiliar with clinical trial concepts often requires more creative methods to ensure a sufficient level of comprehension.

One such creative method to support these populations in understanding their rights and responsibilities when participating in a clinical trial is a multi-media educational tool, a "Speaking Book" entitled 'What it means to be part of a Clinical Trial'. Clinical trials are the gold standard method for collecting safety and efficacy data for health interventions. The Speaking Book (SB) is a richly illustrated book designed to enhance knowledge and understanding of what clinical trials are, how they are conducted, and the rights and responsibilities of participants in a clinical trial. The SB consists of sixteen pages and sixteen corresponding buttons. The text on each page describes one topic around the participation in clinical trials and can be read aloud in English by a sound device within the book, which can be activated by pushing the corresponding button. Each monologue lasts less than a minute. The content of this particular book was reviewed by the World Medical Association to ensure alignment with the principles of the Declaration of Helsinki [1]; by the South African Medical Association to ensure the clinical relevance; and by the Steve Biko Centre of Bioethics to ensure that the rights of human research subjects were addressed. The book can be used by researchers to provide general education to potential clinical trial participants. In a pilot study of 52 participants working in a mass catering company conducted in South Africa [18], the SB was evaluated for efficacy in knowledge uptake and ease of use. The results of this pilot study indicate that incorporating the SB into the consent process increases the level of knowledge of clinical trials among study participants. The study also showed that the participants perceived the educational tool as easy to use.

In order to obtain information about the efficacy of the SB in a research setting in Uganda, a clinical trial was conducted in a busy public clinic located within the National Hospital where patients are recruited for clinical trials. The research team sought to provide information about the effectiveness of the SB in the type of environment for which it was designed. The team also assessed the acceptability of the SB by research participants and health professionals working on clinical trials.

\section{Methods}

The study was reviewed and approved by the Joint Clinical Research Centre (JCRC) Ethics Committee and by the Uganda National Council for Science and Technologym (UNCST). Written consent was obtained from each participant and the ethics committee approved this procedure. The clinical trial is registered with the Pan African Clinical Trials Registry, trial number PACTR201307000574378.

This study was a randomized, controlled clinical trial design comprising 2 groups, each of approximately 100 adult (older than 18 years) participants, in a research site in Kampala, Uganda. Patients attending a health clinic in Kampala were invited to participate in the study by a site research assistant. Those consenting to participate and who could understand and read English (as assessed by a literacy test) were randomized sequentially according to pre-allocated group assignments in blocks of 4 to either the control group or the SB group. Both groups took part in a standard clinical trial information session and participants were assessed immediately afterward using a written 22-item knowledge assessment that was developed by the study team based on the information covered during the session. The total score was calculated as the percentage of correct answers. The assessment addressed the nature of clinical trials, and the rights and responsibilities of participants in clinical trials. After the initial information session and assessment of knowledge, the participants in the SB group were provided instructions on the use of the SB, received a copy of the SB to take home and were encouraged to listen to it as may time they wished to as well to invite other people listen to it. After one week, participants in both groups were re-assessed using the same tool to determine retention of knowledge. Participants in the SB group were also asked a set of additional qualitative questions about their experiences with the SB. Participants in both groups were given approximately $\$ 3$ to cover transport costs on each of the 2 days.

In a separate qualitative evaluation, ten health professionals employed in the same research clinic, but not part of the study, were given the book to listen to and were asked to respond to a brief survey about their perceptions of informed consent, and the efficacy and acceptability of using the SB as part of the consent process.

The mean pre- and post-test score difference was assessed by trial arm using an unpaired t-test of proportions. Qualitative data was summarized using tabulations. Data was analyzed using SAS version 9.2.

\section{Results}

A total of 201 participants were randomized on this trial, including 100 participants in the SB group and 101 in the control group. Ninetyone $(90 \%)$ participants in the control group and $100(100 \%)$ in the Speaking Book group completed both the initial and follow-up tests. The average age of participants was 38 years, 53\% were female and $67 \%$ were employed. Forty (20\%) participants reported they had been invited to participate in a clinical trial, including thirty-nine (19\%) who reported they had participated previously in a clinical trial. The demographic characteristics of study participants in the two arms were similar (Table 1), though there was a trend toward higher education level in the control group.

\begin{tabular}{|l|l|l|l|}
\hline Variable & SB n=100 & $\begin{array}{l}\text { Control } \\
\mathbf{n = 1 0 1}\end{array}$ & p-value \\
\hline Gender & $\mathrm{N}(\%)$ & $\mathrm{N}(\%)$ & \\
\hline Female & $55(55)$ & $51(50.5)$ & 0.52 \\
\hline Educational Level & & & 0.10 \\
\hline Primary & $1(1)$ & $6(5.9)$ & \\
\hline S1-S4 & $48(48)$ & $36(35.6)$ & \\
\hline S5-S7 & $25(25)$ & $25(24.7)$ & \\
\hline Tertiary & $26(26)$ & $34(33.7)$ & \\
\hline Employment & & & 0.49 \\
\hline Employed & $69(69)$ & $65(64.4)$ & \\
\hline Ever asked to participate in a Clinical Trial? & & & 0.50 \\
\hline No & $82(82)$ & $79(78.2)$ & \\
\hline Ever participated in a Clinical Trial? & & & 0.53 \\
\hline No & $82(82)$ & $80(79.2)$ & \\
\hline Age (yrs) & & & \\
\hline Mean (SD) & $37.8(8.6)$ & $37.8(11.5)$ & 0.97 \\
\hline
\end{tabular}

SB: Speaking book; S: secondary; SD: standard deviation.

Table 1: Demographic characteristics of study participants by study arm. 
The mean score for the first assessment was $76.5 \%$ in the control group and $71.7 \%$ in the SB group, which was similar (Table 2). The change in proportion of correct responses from test 1 to test 2 was $2.7 \%$ (95\%CI 0.3-5.0) for the control group and 11.6\% (95\%CI 9.3-13.7) for the SB group, which was statistically significant $(p<0.0001)$. The allocation group was the only variable associated with significance for knowledge increase, measured by proportional score difference; there was no association between knowledge change and other variables such as demographic characteristics, educational level, or previous exposure to clinical trials.

We reviewed item-level responses to the knowledge assessment to determine if there were any trends in knowledge uptake or retention by trial arm. In the intervention arm, there were improvements of greater than $10 \%$ from pre-intervention to post intervention in the proportion responding correctly for 11 of $22(50 \%)$ the assessment items, whereas in the control arm, there were improvements of this same magnitude in only $2(9 \%)$ questionnaire items. Among intervention participants, there were no items with a decrease in proportion responding correctly between the assessments; however, in the control group there was a decrease in proportion of correct responses for 7 of 22 (32\%) assessment items.

All participants in the intervention group were asked questions about their experience with the SB. Almost all participants (99\%) liked the illustrations and found the book easy to use (98\%). Most participants (96\%) heard the spoken voice clearly and 98\% reported understanding the content. Almost all participants (99\%) indicated that members of their community would understand the content if given the speaking book to use. Seventy-two percent of participants reported showing the book to others. On average, participants showed the book to 8 other people in their homes, workplace, church, mosque, clinic or hospital. Most participants (93\%) reported that after listening to the speaking book, they would, in principle, be willing to participate in a clinical trial. Table 3 summarizes the responses given by participants in the SB group.

Interviews were conducted with ten health professionals to assess their perceptions of the potential efficacy, acceptability and use of the SB. The average age of the health professionals interviewed was 31.6 years and they had been working in their current position for an average of 3.8 years. Of the ten health professionals surveyed, seven (70\%) thought that their current consent process at their clinic provided participants with sufficient understanding to sign an informed consent before entering a clinical trial. Most (90\%) thought that participants in clinical trials are aware of their role and responsibilities prior to signing the informed consent form. Four (40\%) thought the person who explains the information sheet and consent form to the patient does not have enough time to make sure that the patient completely understands all the information. Nine (90\%) thought that the consent process would be easier if patients were asked to read the SB first on their own. Seven (70\%) thought that participants take study drug as prescribed and inform the study staff about any additional drugs used. Of the health professionals who thought participants do not take study drug as prescribed (30\%), all thought that the SB would help in explaining the importance of this to them. Five (50\%) of the health professional respondents reported that they had been asked about the term "placebo" during the consent process. Most (80\%) of these thought they understood the term placebo well enough to explain it. Six (60\%) of all health professionals interviewed thought the SB explained the concept sufficiently. All ten (100\%) interviewed reported that they usually told patients that they can quit participation in the trial at any time, and nine (90\%) thought the SB addressed this issue adequately. Three (30\%) thought that the SB contained all the necessary information while seven (70\%) thought the SB contained most but not all of the information necessary to make a decision about participating. Almost all (90\%) thought that each participant should be given a SB to take home before agreeing to participate in a clinical trial, and all ten (100\%) thought the SB would assist participants better than a brochure when screening or informing them about a clinical trial (Table 4).

\section{Discussion}

In settings with patients unfamiliar with clinical trial concepts, innovative techniques can improve knowledge acquisition and

\begin{tabular}{|c|c|c|c|c|}
\hline Group & Test 1 Mean & Test 2 Mean & Mean of Score Difference & t-score \\
\hline Control & $76.5 \%$ & $79.2 \%$ & $2.7 \%$ & $11.6 \%$ \\
\hline Speaking Book & $71.7 \%$ & $83.3 \%$ & -5.3 \\
\hline
\end{tabular}

Table 2: Knowledge test scores (proportion of correct responses) by group.

\section{Question}

Did you like the pictures and drawings?

Did you find the book easy to use?

Could you hear the person talking to you clearly?

Did you understand all the information that she told you in the book?

Do you think members of your church, community, and township will understand what a clinical trial is, if they were given this book to listen to?

Did you show the book to anyone else in your community?

Did you show the book to anyone else at Church/Mosque?

Did you show the book to anyone at work?

Did you show the book to anyone in your family?

Did you show the book to anyone at the clinic or hospital?

Did you show the book to someone anywhere else?

After listening to the information and the story in the book would you ever be willing to be in a clinical trial?

\begin{tabular}{|c|c|c|}
\hline Yes Total (mean) & No Total (mean) & If yes, how many? Total (mean) \\
\hline $99(99)$ & $1(1)$ & \\
\hline $98(98)$ & $2(2)$ & \\
\hline $96(96)$ & $4(4)$ & \\
\hline $98(98)$ & $2(2)$ & \\
\hline $99(99)$ & $1(1)$ & \\
\hline $72(72)$ & $28(28)$ & $190(1.9)$ \\
\hline $54(54)$ & $46(46)$ & $144(1.4)$ \\
\hline $41(41)$ & $59(59)$ & $291(2.9)$ \\
\hline $66(66)$ & $34(34)$ & $161(1.6)$ \\
\hline $47(47)$ & $53(53)$ & $18(0.2)$ \\
\hline $9(9)$ & $91(91)$ & \\
\hline $93(93)$ & $7(7)$ & \\
\hline
\end{tabular}

Table 3: Summary of participant responses to questions about the Speaking Book. 


\section{Question}

Do you think that the consent process at your clinic now is enough for the participants to understand the consent forms provided and the details of the trial?

In general, do you think that participants in clinical trials are aware of their medical responsibilities prior to signing the consent form?

Do your patients understand that they should inform the doctor or nurses about any other medication that they take before or during the trial, even from a pharmacy or a traditional healer?

Do you think the participants in a clinical trial take their medication exactly as they are told to do?

If NO, do you think the book can help you explain the importance of this to them?

Do patients ever ask you what a placebo is during the consent process?

If YES, do you think that you know about a placebo well enough to explain it properly?

Do you think the speaking book explains the concept of a placebo enough?

Do you usually tell the patient that they can stop the clinical trial at any time?

Does the book tell the patient clearly enough that they can stop the clinical trial at any time?

Do you think that the person who explains the information sheet and consent form to the patient has enough time to make sure that the patient completely understands all the information?

Do you think that the consent process would be easier if the patient was asked to read the book first on their own?

Do you think that the information in the book gives all the information needed to make a decision about participating?

Do you think the book should include any other information we have forgotten?

At what time do you think that the books should be given to the new person applying for the trial?

At time of first visit to the research clinic

At time of first talk about clinical trial

Do you think that each participant should be given a speaking book to take home before agreeing to participate in a clinical trial?

If you were going through screening or informing a patient about a clinical trial, in addition to normal practices which do you think would help a participant more?

Speaking Book

Brochure
Yes $n(\%)$ No $n(\%) \quad$ No response $n(\%)$

$7(70)$

$9(90)$

$7(70)$

$7(70)$

$3(30)$

5(50) 4(40)

4(40) 1(10)

$6(60) \quad 4(40)$

10(100) $0(0)$

$9(90) \quad 1(10)$

$6(60) \quad 4(40)$

$9(90) \quad 1(10)$

3(30) $0(0)$

$3(30) \quad 7(70)$

$4(40)$

9(90)

$1(10)$

$10(100)$

$0(0)$

Table 4: Summary of Health Professionals responses to questions about the Speaking Book

retention in order for individuals to make a more informed choice about participation in clinical trials. Participants who had instruction in the use of the SB and used it for one week had a larger improvement in knowledge assessment score compared to those who had no access to this tool. Our data is in contrast to a meta-analysis by Flory and Emanuel [13].

The SB seems to be a valuable tool in improving patients understanding of clinical trials and their rights and responsibilities associated with participation in a trial. The qualitative assessment of the intervention group showed that participants who were instructed in the use of the SB and brought it home for a week found it useful and shared it extensively with friends, family, work colleagues and other associates, thereby increasing the value of the book as an educational tool. This allowed patients to discuss the ethical aspects of clinical trials with others whose opinions they valued.

A structured questionnaire was used with a limited pool of health professionals who viewed the SB as a useful tool for increasing the capacity of patients to make an informed decision regarding participation in a clinical trial.

One limitation of our study was that the participants included only those who spoke and understood English since the SB was not translated into local languages. Therefore, participants likely had a higher educational status than the average for the clinic. In the metaanalysis [13], research participants with higher education status were more likely to have better understanding. Nonetheless, having a group of participants capable of taking the test represented an appropriate first group in whom to test the intervention. The investigators also noted that despite the randomization the control arm had slightly higher education level, though of marginal significance $(\mathrm{p}=0.10)$, and therefore the use of the SB could have had an even higher impact on the absolute score change if groups had a more similar level of education. The fact that a differential improvement in knowledge was identified between the study groups suggests that the SB might demonstrate an even greater improvement in knowledge among a less literate population. Further studies with use of the tool in the local language such that participants with lower educational status could be included would be warranted.

A disadvantage of using the SB to pass information on clinical trials is that it requires a two-visit procedure with increase in study costs and potential for loss to follow up in between the visits. However in our study all participants in the SB arm (as compared to $90 \%$ in the control arm), returned for the follow up visit after the week, possibly as the result of learning the importance of participating clinical trials; in addition most of the participants showed the book to an average of 8 other people in their homes, contributing to the sensitization of the general population on clinical trials.

In summary, the use of a SB multi-media tool for one week after a standard explanation of clinical trials was able to increase comprehension scores significantly compared to participants who received only one educational session. The SB is an introductory tool that can be used to inform patients on topics common to all clinical trials and may be a valuable adjunctive instrument for use among potential research participants to improve understanding of clinical trials and make an informed decision during the consent process.

\section{Acknowledgements}

This study was made possible through a grant from Pfizer Inc. This project has been funded in whole or in part with federal funds from the National Cancer 
Citation: Castelnuovo B, Newell K, Manabe YC, Robertson G (2014) Multi-media Educational Tool Increases Knowledge of Clinical Trials in Uganda. J Clin Res Bioeth 5: 165. doi:10.4172/2155-9627.1000165

Institute, National Institutes of Health, under Contract No. HHSN261200800001E. The content of this publication does not necessarily reflect the views or policies of the Department of Health and Human Services, nor does mention of trade names, commercial products, or organizations imply endorsement by the U.S. Government. This research was supported [in part] by the National Institute of Allergy and Infectious Diseases. The authors would like to acknowledge and thank Margaret Denty, Study Coordinator at the Infectious Diseases Institute of Makerere University, Kampala for her hard work in the conduct of this study and Søren Rasmussen for his valuable support.

\section{References}

1. World medical Association (1964) Declaration of Helsinki - Ethical Principles for Medical Research Involving Human Subjects.

2. Byrne DJ, Napier A, Cuschieri A (1988) How informed is signed consent? $\mathrm{Br}$ Med J (Clin Res Ed) 296: 839-840.

3. Lavelle-Jones C, Byrne DJ, Rice P, Cuschieri A (1993) Factors affecting quality of informed consent. BMJ 306: 885-890.

4. Joubert G, Steinberg H, van der Ryst E, Chikobvu P (2003) Consent for participation in the Bloemfontein vitamin A trial: how informed and voluntary? Am J Public Health 93: 582-584.

5. Molyneux CS, Peshu N, Marsh K (2004) Understanding of informed consent in a low-income setting: three case studies from the Kenyan Coast. Soc Sci Med 59: 2547-2559.

6. Abdool Karim Q, Abdool Karim SS, Coovadia HM, Susser M (1998) Informed consent for HIV testing in a South African hospital: is it truly informed and truly voluntary? Am J Public Health 88: 637-640.

7. Agre P, Kurtz RC, Krauss BJ (1994) A randomized trial using videotape to present consent information for colonoscopy. Gastrointest Endosc 40: 271-276.

8. Ives NJ, Troop M, Waters A, Davies S, Higgs C, et al. (2001) Does an HIV clinical trial information booklet improve patient knowledge and understanding of HIV clinical trials? HIV Med 2: 241-249.
9. Llewellyn-Thomas HA, Thiel EC, Sem FW, Woermke DE (1995) Presenting clinical trial information: a comparison of methods. Patient Educ Couns 25 97-107.

10. Bygrave H, Kranzer K, Hilderbrand K, Jouquet G, Goemaere E, et al. (2011) Renal safety of a tenofovir-containing first line regimen: experience from an antiretroviral cohort in rural Lesotho. PLoS One 6: e17609.

11. Ryan RE, Prictor MJ, McLaughlin KJ, Hill SJ (2008) Audio-visual presentation of information for informed consent for participation in clinical trials. Cochrane Database Syst Rev: CD003717.

12. Tamariz L, Palacio A, Robert M, Marcus EN (2013) Improving the informed consent process for research subjects with low literacy: a systematic review. $J$ Gen Intern Med 28: 121-126.

13. Flory J, Emanuel E (2004) Interventions to improve research participants' understanding in informed consent for research: a systematic review. JAMA 292: 1593-1601.

14. Dunn LB, Lindamer LA, Palmer BW, Golshan S, Schneiderman LJ, et al. (2002) Improving understanding of research consent in middle-aged and elderly patients with psychotic disorders. Am J Geriatr Psychiatry 10: 142-150.

15. Hoffner B, Bauer-Wu S, Hitchcock-Bryan S, Powell M, Wolanski A, et al. (2012) "Entering a Clinical Trial: Is it Right for You?": a randomized study of The Clinical Trials Video and its impact on the informed consent process. Cancer 118: $1877-1883$.

16. Sengupta S, Lo B, Strauss RP, Eron J, Gifford AL (2011) Pilot study demonstrating effectiveness of targeted education to improve informed consent understanding in AIDS clinical trials. AIDS Care 23: 1382-1391.

17. Joseph P, Schackman BR, Horwitz R, Nerette S, Verdier RI, et al. (2006) The use of an educational video during informed consent in an HIV clinical trial in Haiti. J Acquir Immune Defic Syndr 42: 588-591.

18. Dhai A, Etheredge H, Cleaton-Jones $P$ (2010) A pilot study evaluating an intervention designed to raise awareness of clinical trials among potential participants in the developing world. J Med Ethics 36: 238-242. 